\title{
Variáveis que influenciam no uso da bicicleta e as crenças da teoria do comportamento planejado
}

\author{
Mariana Oliveira da Silveira ${ }^{1}$, Maria Leonor Alves Maia²
}

\begin{abstract}
Resumo: Pesquisas sobre bicicleta que trabalham o componente 'comportamento' vêm ganhando destaque no intuito de entender as barreiras e os motivadores ao uso deste modo de transporte e ajudam a planejar melhores formas de estimular seu uso. O objetivo deste artigo é apresentar um panorama das variáveis que influenciam no uso da bicicleta e classificá-las junto às crenças e constructos da Teoria do Comportamento Planejado (TCP). Este panorama foi baseado em pesquisa da base SCOPUS e em artigos de anais e periódicos, teses e dissertações nacionais, o qual, além de enumerar fatores que influenciam no uso da bicicleta, servirá de apoio às pesquisas que pretendem aplicar a Teoria do Comportamento Planejado na avaliação de comportamentos de uso deste modo de transporte. As variáveis 'tempo de deslocamento com o uso da bicicleta', 'condições meteorológicas', 'segurança do trânsito', 'infraestrutura cicloviária' e 'comportamento no trânsito entre motoristas e ciclistas' foram as que mais se destacaram, de acordo com o levantamento bibliográfico, na interferência de intenção e de comportamento de uso da bicicleta.
\end{abstract}

Palavras-chave: bicicleta, comportamento, teoria do comportamento planejado.

\begin{abstract}
The behavior component related to the use of bicycle is gaining prominence in the non motorized transport research. It helps understanding the barriers and motivations that induce the use of this modal. The objective of this article is to present an overview from the national and international literature about the main variables that influence cycling and categorize them along to beliefs and constructs of the Theory of Planned Behavior (TPB). This overview was based on international researches from SCOPUS. Besides presenting the beliefs that influence the use of cycling, it intend to support researches that intend to apply the Theory of Planned Behavior in the evaluation of behaviors regarding the use of bicycles. The variables 'time offset with the use of the bicycle', 'weather', 'traffic safety', 'cycling infrastructure' and 'behavior in traffic between motorists and cyclists' were the ones that stood out, according to the survey literature, the interference of intent and behavior of bicycle use.
\end{abstract}

Keywords: bicycle; behavior; theory of planned behavior (TPB).

\section{INTRODUÇÃO}

A imagem comumente equivocada da bicicleta em certos países a exemplo do Brasil, caracterizada por ser modal de transporte de pessoa de baixa renda e de ser modal desvalorizado entre todos os modais (com relação à acessibilidade e infraestruturas para seu uso), é reflexo de como a bicicleta tem sido utilizada na maioria das cidades ocidentais, da própria organização social e seus valores. O símbolo de status dado ao automóvel e a desvalorização da bicicleta enquanto um modal de transporte são fatores que impedem a maior utilização da bicicleta. Refinar o entendimento de como se percebe a bicicleta pode dar subsídios para mudar essa imagem da bicicleta e propiciar a utilização da mesma, não só para o lazer, mas também para uso frequente (viagens para escola e/ou trabalho). Dar atenção ao ciclista verificando suas barreiras e motivações ao uso da bicicleta é facilitar a sua introdução no sistema de transporte de qualquer cidade. A imagem da bicicleta inclui aspectos cognitivos, como por exemplo, a praticidade, mas também afetivos, como o prazer de andar de bicicleta. Isso fornece um

\footnotetext{
1 Mariana Oliveira da Silveira, Programa de Pós-Graduação em Engenharia Civil.Universidade Federal de Pernambuco - UFPE, Recife PE. (maridasilveira@gmail.com)

2 Maria Leonor Alves Maia, Programa de Pós-Graduação em Engenharia Civil. Universidade Federal de Pernambuco - UFPE. (nonamaia@gmail.com)

Manuscrito recebido em 18/09/2014 e aprovado para publicação em $11 / 12 / 2014$.

Este artigo é parte de TRANSPORTES v. 23, n. 1, 2015. ISSN: 2237-1346 (online). DOI:10.4237/transportes.v23i1.848.
}

amplo leque de possibilidades de intervenção para aumentar o seu uso (DELABRIDA, 2004).

Vasconcelos (2000) ressalta que recorrer às experiências feitas na Europa, por exemplo, para o uso da bicicleta, pode trazer boas ideias, mas, se não se considerar que a percepção dos europeus sobre a bicicleta normalmente é de boa aceitação, as conclusões podem ser enganadoras e os resultados podem ser frustrantes. É preciso levar em consideração essa diferença de percepção que há entre os europeus e os brasileiros, por exemplo. Estudar a imagem da bicicleta pode nos auxiliar a identificar que aspectos dessa percepção são semelhantes e que aspectos são distintos e nos auxiliar nas intervenções que favoreçam o uso da bicicleta.

Franco (2011) destaca que, se a mobilidade urbana depende da fluidez do sistema de trânsito, é necessário que a imagem da bicicleta seja promovida a um status de modal para uso frequente e não de um veículo apenas para o lazer. Pesquisas de comportamento das populações a serem alcançadas como potenciais usuárias da bicicleta são importantes para influenciar novos usuários.

O objetivo deste artigo é apresentar um panorama das variáveis que influenciam no uso da bicicleta e classificálas junto às crenças e constructos da Teoria do Comportamento Planejado (TCP). A relação das variáveis com constructos e crenças, as quais de acordo com a TCP podem ser comportamentais, normativas ou de controle, facilitará na enumeração das variáveis que podem influenciar na intenção de se usar a bicicleta nas cidades e consequentemente poderá apoiar às pesquisas que pretendem aplicar a Teoria do Comportamento Planejado na avaliação de comportamentos de uso da bicicleta. 


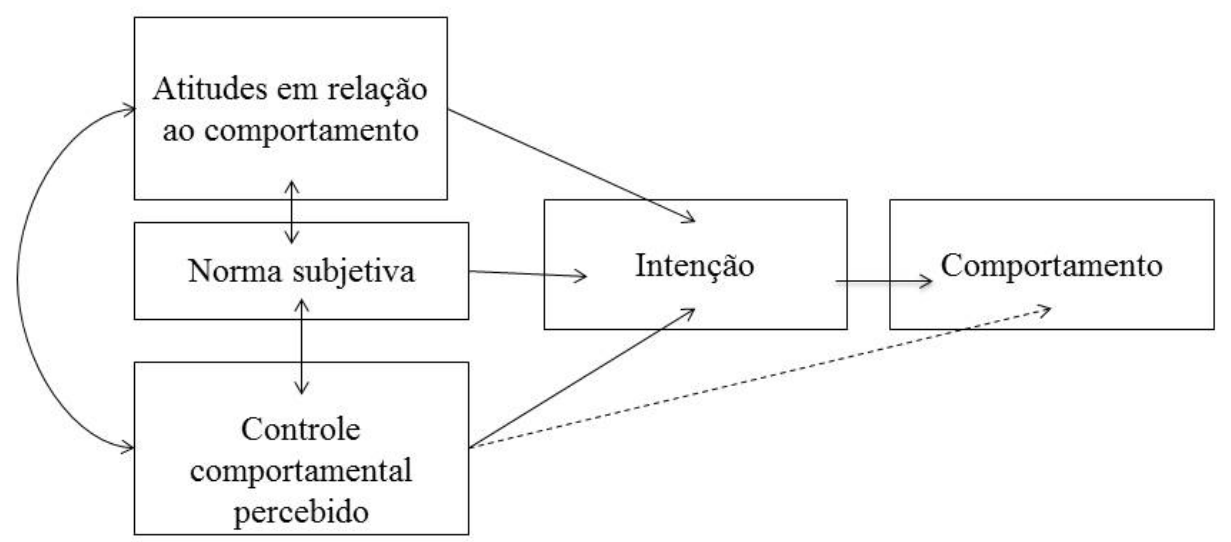

Figura 1. Teoria do Comportamento Planejado

Fonte: Ajzen (1991), adaptada pelas autoras (2013)

A Teoria do Comportamento Planejado (TCP) vem sendo utilizada em pesquisas recentes sobre bicicleta que trabalham o componente 'comportamento', principalmente no âmbito internacional. Num levantamento feito na base SCOPUS (base bibliográfica de cobertura internacional da Editora Elsevier), do ano 2000 ao 2013, num universo de 79 pesquisas que relacionam comportamento com bicicleta, 15 delas trabalharam modelos e teorias. Dessas 15 pesquisas, 7 delas utilizaram a TCP.

Esse artigo segue estruturado em 5 seções além da introdução. A seção 2 apresenta a Teoria do Comportamento Planejado (TCP). Na seção 3 é apresentado o levantamento de variáveis que influenciam no uso da bicicleta no Brasil. Na seção 4 é apresentado o levantamento de variáveis que influenciam no uso da bicicleta no exterior. Na seção 5 é apresentada a relação das variáveis que influenciam no uso da bicicleta com as crenças da Teoria do Comportamento Planejado. E por fim, na última seção, tecem-se comentários conclusivos ressaltando a importância do levantamento de variáveis que influenciam no uso da bicicleta, principalmente para pesquisas que pretendem aplicar a Teoria do Comportamento Planejado para identificar as atitudes, as normas subjetivas, os controles comportamentais percebidos e intenções comportamentais sobre este uso.

\section{TEORIA DO COMPORTAMENTO PLANEJADO}

A Teoria do Comportamento Planejado (TCP) consiste em um modelo de múltiplos atributos através do qual a intenção comportamental - o mais próximo que se pode chegar do comportamento - é determinada por três constructos: (1) atitudes em relação ao comportamento, (2) norma subjetiva e o (3) controle comportamental percebido (AJZEN, 1985) (ver Figura 1). A importância desses constructos na previsão de intenções é encontrada em vários casos de comportamentos e situações, sendo, em alguns deles, apenas as atitudes que possuem impacto relevante nas intenções, em outros, atitudes e controle podem ser suficientes, e, ainda, em outros casos, os três determinantes serão relevantes (FLEISCHFRESSER, 2005). A TCP tem como objetivo compreender e predizer os comportamentos sociais, ou seja, busca prever as intenções comportamentais que precedem e condicionam os comportamentos reais, em vez de conhecer unicamente as atitudes, as normas subjetivas e as crenças do indivíduo.
O termo crença está relacionado às variáveis ou fatores que influenciam na intenção comportamental do indivíduo. Este termo é usado na Teoria do Comportamento Planejado e por isto será considerado em toda a explanação deste artigo.

Os três constructos da Teoria do Comportamento Planejado são assim explicados:

De acordo com Ajzen (1991) a ATITUDE se refere ao grau de avaliação pessoal, que pode ser favorável ou desfavorável com relação ao comportamento em questão. Pode-se considerar atitude como um conceito multifacetado, que consiste em três componentes: (1) cognitivo (conhecimentos, crenças); (2) afetivo (gostos, preferências) e (3) comportamental. Sua mensuração envolve uma série de variáveis internas ao indivíduo, sustentadas pelos três componentes citados que estão em constante inter-relação (AJZEN e FISHBEIN, 2000).

O constructo NORMA SUBJETIVA refere-se, por sua vez, à percepção das expectativas que indivíduos ou grupos têm a respeito de seus comportamentos. São as influências causadas pela pressão social de pais, amigos, grupos de referência, cultura, opinião pública e instituições sobre o comportamento dos indivíduos. Assume-se que as crenças normativas combinadas com a motivação pessoal em agir em conformidade com as expectativas sociais determinam a norma subjetiva que irá prevalecer (PEIXOTO, 2007).

Por fim, para Ajzen (1991) o CONTROLE PERCEBIDO sobre o comportamento é definido como a crença do indivíduo sobre o grau de facilidade ou de dificuldade para desempenhar um determinado comportamento. Para as pessoas, normalmente os comportamentos são considerados como metas sujeitas a interferências e incertezas e o controle percebido sobre o comportamento serve para verificar até que ponto o decisor leva em consideração problemas pessoais e fatores situacionais que interferem positiva ou negativamente no desenvolvimento da ação.

O constructo controle percebido ajuda a explicar por que as intenções sozinhas, em algumas situações, não são capazes de predizer o comportamento. A razão para que o constructo controle percebido tenha uma ligação direta com o comportamento é que quando os indivíduos sentem que possuem grande controle sobre o comportamento e as intenções comportamentais isoladamente, são capazes de prever o comportamento (ver Figura 1) (MOURA et.al. 2012). 
De acordo com Moutinho e Roazzi (2010) achados evidenciam que investigações norteadas pela Teoria do Comportamento Planejado podem colaborar para a definição de políticas públicas e viabilizar campanhas educativas. Além disso, destacam que esta teoria é particularmente útil e crucial em circunstâncias/projetos/programas nos quais o comportamento das pessoas precisa ser modificado, como exemplo temos algumas circunstâncias de mau comportamento no trânsito.

Heidemann, Araújo e Veit (2012) defendem a Teoria do Comportamento Planejado como um referencial teóricometodológico potencialmente útil para pesquisas de ensino de ciências que envolvam aferição de atitudes. Além disso, argumentam que a Teoria do Comportamento Planejado tem potencial de amparar pesquisadores na confecção de instrumentos para mensuração de atitudes, na interpretação de seus resultados e no planejamento de programas de intervenção que aumentem as chances de consecução de seus objetivos.

De acordo com Ajzen (2006) intervenções com o intuito de mudar o comportamento podem ser dirigidas a um ou mais de seus determinantes: as atitudes, as normas subjetivas ou o controle comportamental percebido. Alterações nesses fatores devem provocar mudanças nas intenções comportamentais e, consequentemente, devem provocar modificações no comportamento. No entanto, o sujeito que planeja a intervenção deve considerar se há espaço para a mudança no constructo selecionado. Se por exemplo, uma pesquisa já mostra que a atitude dos indivíduos alvo é altamente favorável ao comportamento, é pouco provável que uma intervenção destinada a tornar as atitudes dos sujeitos ainda mais favoráveis vá obter bons resultados. Nesse caso, deve-se selecionar outro público alvo que ofereça maior possibilidade de modificações.

\subsection{Limites das pesquisas que envolvem a Teoria do Comportamento Planejado}

No caso de pesquisas no Brasil, de acordo com Rodrigues (2007), o principal problema das pesquisas que usam a Teoria do Comportamento Planejado em sua metodologia é o fato de que a maioria dos estudos encontrados são muito antigos, e mesmo os atuais não refletem os achados recentes da literatura. Além disso, na área de atitudes, seu aspecto instrumental e expressivo é geralmente ignorado. Apesar dos determinantes de intenção e a relação atitude-comportamento serem dois objetos de estudo extremamente analisados na literatura nacional e de vital importância para o comportamento humano, no Brasil essa área tem recebido pouca atenção, e os achados relativos à percepção de controle sobre o comportamento são praticamente inexistentes.

Quando observamos pesquisas do exterior, como algumas levantadas na base SCOPUS de 2000 a 2013 referentes ao uso da bicicleta, observa-se que a Teoria do Comportamento Planejado vem sendo mais trabalhada que no Brasil, por exemplo, e se adequa bem no intuito de identificar as intenções de comportamento. O único limite observado, nessas pesquisas, é o número de constructos da teoria, o qual normalmente é inferior aos necessários para compreender e predizer as intenções comportamentais que precedem e condicionam os comportamentos reais. No entanto, este limite vem sendo resolvido com a inclusão de novos constructos, medida inclusive indicada pelos autores da teoria, Ajzen e Fishbein (1980).

Temos como exemplos desses novos constructos as variáveis de norma descritiva (que são relacionadas à como os outros se comportam em relação ao comportamento), a força de hábito (inclusive muito trabalhada em pesquisas que abordam o comportamento de uso da bicicleta), e os constructos de natureza sociodemográfica, como idade, sexo, nível socioeconômico, nível educacional, etc.

\section{VARIÁVEIS QUE INFLUENCIAM NO USO DA BICICLETA NO BRASIL}

No intervalo de 2000 a 2013, diversos perfis de usuários da bicicleta e também motivadores e empecilhos ao uso da bicicleta foram levantados em artigos nacionais de anais de congresso e periódicos e em teses e dissertações nacionais e pôde-se verificar que no Brasil há características semelhantes em diferentes lugares, principalmente quanto às limitações ao uso da bicicleta relativas ao perigo no trânsito. Entretanto, diante da diversidade de características nas cidades do país, é necessário conhecer e estudar as especificidades de cada lugar, para que a incorporação da bicicleta no sistema de transporte da cidade seja de forma adequada.

Dentre as 16 pesquisas levantadas no Brasil, 8 deram destaque a 8 motivadores ao uso da bicicleta. $\mathrm{O}$ motivador 'andar de bicicleta é bom para a saúde', mais citado entre as variáveis levantadas, ganha destaque em cinco cidades: três de pequeno porte populacional (São Carlos, Rio Claro e São José do Rio Preto em São Paulo), uma de médio porte populacional (Aracajú-SE) e apenas em uma de grande porte populacional (Salvador-BA). Os portes populacionais estão de acordo com o censo IBGE 2010.

Outros dois motivadores ao uso da bicicleta que se destacaram foram 'baixo custo da bicicleta' e 'redução no tempo de deslocamento' adquirido com o uso da bicicleta. Cada um desses motivadores foi citado em três cidades, com destaque para Salvador-BA, que dentre as cidades pesquisadas foi a que mais demostrou motivadores ao uso da bicicleta.

Duas variáveis que foram citadas como motivadores ao uso da bicicleta, mas que apresentam baixa frequência de citação, pois foram citadas apenas uma vez, foram: 'transporte coletivo insuficiente no bairro', citada na cidade de Salvador-BA; e 'opinião da família quanto ao uso da bicicleta', citada na cidade de Curitiba-PR.

Na Figura 2 a seguir, há um resumo do levantamento das variáveis que motivam o uso da bicicleta no Brasil. As variáveis levantadas nas pesquisas nacionais que interferem como motivadoras ao uso da bicicleta foram: 'praticidade de uso da bicicleta', 'bem estar em andar de bicicleta', 'andar de bicicleta faz bem para a saúde', 'baixo custo da bicicleta', 'redução de tempo de deslocamento com o uso da bicicleta', 'transporte coletivo insuficiente no bairro', 'conscientização com a questão ambiental', e 'opinião da família quanto ao uso da bicicleta'.

Ainda sobre o que demonstra a Figura 2, todas as variáveis que motivam o uso da bicicleta estão correlacionadas com cada pesquisa levantada, neste caso foram 8 pesquisas, sendo cada variável mencionada em uma ou mais 


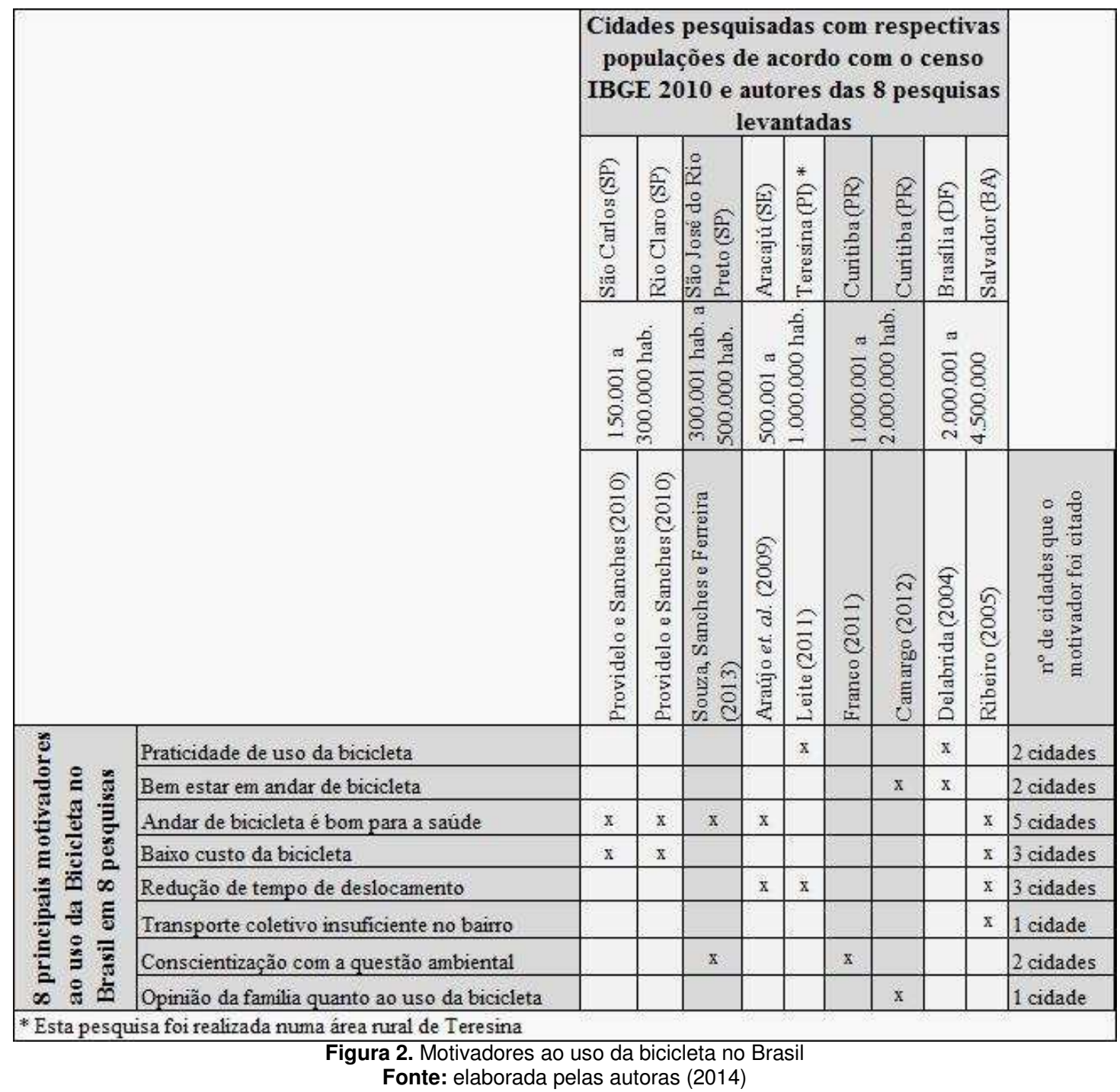

cidades. Por fim, na última coluna da Figura 2, há uma relação do número de cidades que cada motivador ao uso da bicicleta foi citado, por exemplo, 'o baixo custo da bicicleta' foi citado como motivador em três cidades, São Carlos-SP, Rio Claro-SP e Salvador-BA.

Com relação às variáveis de empecilho ao uso da bicicleta, das 16 pesquisas levantadas, 15 mencionaram algum empecilho dos 22 empecilhos identificados. A 'insegurança no trânsito', foi o mais citado e mencionado em quatorze cidades: cinco de pequeno porte populacional (Lorena-SP, Araçatuba-SP, Piracicaba-SP, São José do Rio Preto-SP e Florianópolis-SC), três de médio porte populacional (Aracajú-SE, Santo André-SP e Juiz de Fora-MG) e em seis de grande porte populacional (Salvador-BA, Recife-PE, Curitiba-PR, Brasília-DF, Fortaleza-CE e Belo Horizonte-MG). Os portes populacionais estão de acordo com o censo IBGE 2010.

Outros dois empecilhos ao uso da bicicleta que se destacaram foram 'falta de vias cicláveis', citado em nove cidades (Lorena-SP, Araçatuba-SP, Piracicaba-SP, Santo André-SP, Florianópolis-SC, Recife-PE, Curitiba-PR, Fortaleza-CE e Belo Horizonte-MG) e o 'comportamento no trânsito entre motoristas e ciclistas', citado em sete cidades (Lorena-SP, Piracicaba-SP, Santo André-SP, FlorianópolisSC, Juiz de Fora-MG, Curitiba-PR e Fortaleza-CE). Este comportamento no trânsito entre ciclista e motorista é relativo ao conflito de convivência e desrespeito de espaço viá- rio que ocorre quando ambos (motorista e ciclista) compartilham da mesma faixa de trânsito. Este conflito pode ser ocasionado por parte do motorista, por parte do ciclista ou por ambas as partes. Vale frisar que este tipo de conflito também pode ocorrer entre ciclistas e pedestres, mas esta característica não vem sendo destacada nas pesquisas levantadas.

Houve também um conjunto diverso de variáveis que foram citadas como empecilhos ao uso da bicicleta no Brasil, mas que apresentam baixa frequência de citação. Vários empecilhos foram citados apenas uma vez, tais como: ' falta de bicicletário e vestiário no destino da viagem', citada na cidade de Belo Horizonte-MG; 'desconforto em andar de bicicleta', citada na cidade de Araçatuba-SP; 'falta da integração da bicicleta com outros modais', citada na cidade de Salvador-BA; 'opinião da família quanto ao uso da bicicleta', citada na cidade de Aracajú-SE; 'facilidade para aquisição de motocicletas', citada em área rural da cidade de Teresina-PI; 'vulnerabilidade do ciclista', citada na cidade de Brasília-DF; 'falta de vontade em andar de bicicleta', 'condições meteorológicas', 'poluição causada pelos modais motorizados' e 'alto nível socioeconômico', citadas na cidade de Curitiba-PR e 'sedentarismo', citada na cidade de Belo Horizonte-MG. 
Na Figura 3 a seguir, há um resumo do levantamento das variáveis que desmotivam o uso da bicicleta no Brasil. Como foi feito na Figura 2, todas as variáveis que desmotivam o uso da bicicleta estão correlacionadas com cada pesquisa levantada, neste caso foram 15 pesquisas, sendo cada variável mencionada em uma ou mais cidades. Por fim, na última coluna da Figura 3, há uma relação do número de cidades que cada empecilho ao uso da bicicleta foi citado. Por exemplo, 'falta de vias cicláveis' foi citado como empecilho em nove cidades, Lorena-SP, Araçatuba-SP, Piracicaba-SP, Florianópolis-SC, Santo André-SP, Recife-PE, Curitiba-PR, Fortaleza-CE e Belo Horizonte-MG.

\section{VARIÁVEIS QUE INFLUENCIAM NO USO DA BICICLETA NO EXTERIOR}

No intervalo de 2000 a 2013, diversos perfis de usuários da bicicleta e também motivadores e empecilhos ao uso da bicicleta foram levantados na base SCOPUS e pôdese verificar que no exterior o número de limitações ao uso da bicicleta é normalmente menor que no Brasil, enquanto que o de motivadores é maior. Percebeu-se também que cidades dos EUA e da Europa ganham destaque no rol de pesquisas que objetivam identificar barreiras e motivadores ao uso da bicicleta. Em seguida, cidades da Oceania também ganham destaque, principalmente as situadas na Austrália.

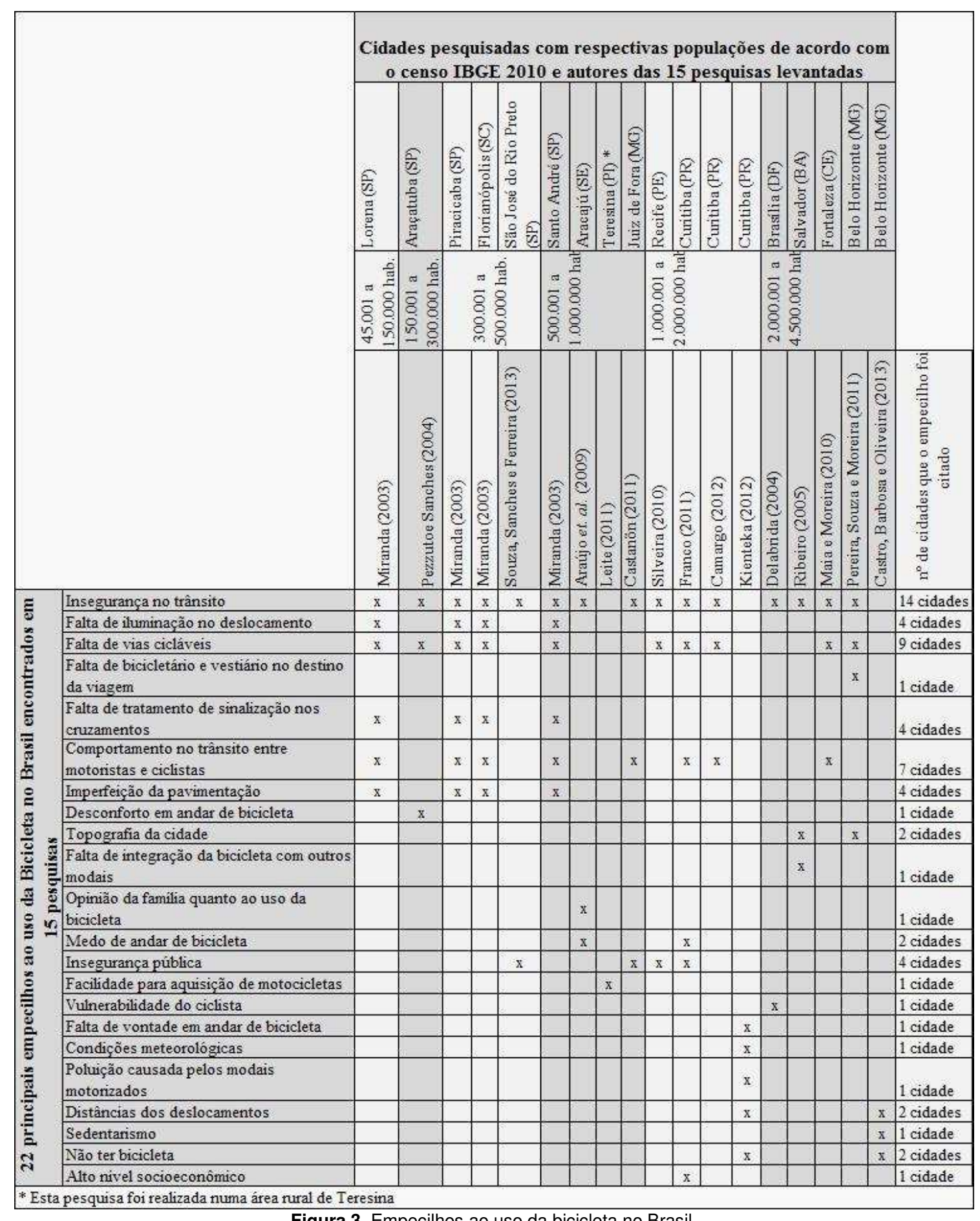

Figura 3. Empecilhos ao uso da bicicleta no Brasil.

Fonte: elaborada pelas autoras (2014) 
No resumo do levantamento das variáveis que influenciam no uso da bicicleta no exterior (ver Figuras 4 e 5) cada variável foi considerada por pesquisa e não por cidade estudada, pois há casos em que a pesquisa considera vários municípios do país, às vezes até todos os municípios, como é o caso, por exemplo, da pesquisa de Vandenbulcke et.al. (2009) e Vandenbulcke et.al. (2011) onde todos os municípios da Bélgica são considerados. Outras observações sobre quais cidades são consideradas por pesquisa estão disponíveis abaixo de cada figura.

Dentre as 34 pesquisas levantadas no exterior, 30 mencionaram 21 motivadores ao uso da bicicleta. O motivador 'acessibilidade e infraestrutura cicloviária', mais citado entre as variáveis levantadas, ganha destaque em treze pesquisas (quatro da Oceania, quatro da Europa, três da América do Norte e duas da Ásia). Os motivadores 'pequenas distâncias entre origem e destino', 'segurança no trânsito', 'condições meteorológicas' e 'medidas de redução de velocidade para motorizados e sinalização bem sucedida' foram outros motivadores mais mencionados, citados em cinco pesquisas cada um (ver Figura 4).

Houve também um conjunto diverso de variáveis que foram citadas como motivadoras ao uso da bicicleta no exterior, mas apresentam baixa frequência de citação. Vários motivadores foram citados apenas uma vez, foram eles: 'ter poucos cruzamentos difíceis no deslocamento', citada apenas na pesquisa de Tin Tin et. al. (2009), realizada na cidade de Taupo (Nova Zelândia); 'opinião de colegas quanto ao uso da bicicleta', citada apenas na pesquisa de Heinen, Maat e Wee (2013), realizada na Holanda e 'praticidade da bicicleta', citada apenas na pesquisa de Heinen, Maat e Wee (2011), também realizada na Holanda (ver Figura 4).

Dentre as 34 pesquisas levantadas no exterior, 15 mencionaram 15 empecilhos ao uso da bicicleta. O empecilho 'condições meteorológicas', mais citado entre as variáveis levantadas, foi mencionado em cinco pesquisas. Esta variável, que também é citada como motivadora ao uso da bicicleta em cinco pesquisas, teve no total das 34 pesquisas 4 citações em comum aos motivadores e empecilhos. Esta característica de ser tanto motivadora quanto empecilho é devido a variação do clima que pode favorecer ou inibir o uso da bicicleta. As pesquisas que esta variável aparece tanto quanto motivadora quanto como empecilho foram realizadas em área rural da Holanda perto das cidades de Gouda e Ede; na cidade de Auckland (Nova Zelândia); e no Canadá (ver Figura 5).

A variável 'falta de infraestrutura cicloviária continuada' foi a segunda mais mencionada entre os empecilhos no exterior, citada em 3 pesquisas: na de Fishman, Washington e Haworth (2012), realizada na cidade de Brisbane (Austrália); na de Wahlgren e Schantz (2012), realizada na cidade de Estocolmo (Suécia) e na de Li et. al. (2012), realizada na cidade de Nanquim (China) (ver Figura 5).

Houve também um conjunto diverso de variáveis que foram citadas como empecilhos ao uso da bicicleta no exterior, mas que apresentam baixa frequência de citação. Vários empecilhos foram citados apenas uma vez, foram eles: ‘imperfeição da pavimentação' e 'velocidade do tráfego motorizado', citadas na pesquisa de Winters et. al. (2011), realizada em Vancouver (Canadá); 'tempo de deslocamento', citada na pesquisa de Engbers e Hendriksen (2010), realizada na Holanda; 'distância dos deslocamentos' e 'necessidade de se carregar bagagens durante a viagem', citadas na pesquisa de Heinen, Maat e Wee (2013), realizada nas cidades holandesas de Delft, Zwolle, Midden-Delfland e Pijnacker-Nootdorp; 'poluição causada pelos modais motorizados', citada na pesquisa de Wahlgren e Schantz (2012), realizada em Estocolmo (Suécia); 'medo de andar de bicicleta', citada na pesquisa de Ahlport et. al. (2008), realizada na Carolina do Norte (EUA) e 'alto nível socioeconômico', citada na pesquisa de Plaut (2005), realizada nos EUA (ver Figura 5). Percebe-se, como dito anteriormente, que o número citado das variáveis no exterior é considerado por pesquisa e não por cidade.

Observa-se que nos levantamentos no âmbito nacional e internacional, a variável 'acessibilidade e infraestrutura cicloviária' se destaca como o segundo maior empecilho dentre as cidades do Brasil e como maior motivador entre as pesquisas do exterior, sendo citado como empecilho no Brasil em nove cidades e como motivador no exterior em treze pesquisas.

\section{VARIÁVEIS QUE INFLUENCIAM NO USO DA BICICLETA E AS CRENÇAS DA TEORIA DO COMPORTAMENTO PLANEJADO}

Crenças desempenham um papel central na Teoria do Comportamento Planejado. Elas são consideradas as bases cognitivas e afetivas para as atitudes, normas subjetivas e para o controle comportamental percebido. Ao medir as crenças, portanto, pode-se, teoricamente, investigar por que as pessoas têm certas atitudes, normas subjetivas e controles comportamentais percebidos.

Ajzen (2005) salienta que as crenças mais facilmente acessíveis na memória das pessoas e as que o indivíduo expressa mais comumente e em maior intensidade são as que determinam seus comportamentos, sendo essas conhecidas como crenças salientes. Algumas crenças podem persistir com o passar do tempo, outras podem enfraquecer ou desaparecer. Além disso, novas crenças podem ser formadas e, dessa forma, as pessoas podem ter um grande número de crenças sobre um dado objeto. Ajzen considera que normalmente o número de crenças salientes é relativamente pequeno, talvez oito ou nove.

De acordo com Moutinho e Roazzi (2010) sugere-se que, num primeiro momento para predizer algum comportamento, sejam levantadas as crenças salientes dos indivíduos alvo da pesquisa. Uma vez que as crenças salientes tenham sido identificadas, um questionário padrão da Teoria do Comportamento Planejado pode ser elaborado, incluindo medidas de crença, atitude, norma subjetiva, percepção de controle comportamental, intenção e comportamento real.

As crenças salientes podem ser levantadas por meio de questões dissertativas ou, de acordo com Ajzen (2005), podem ser formadas como resultado de observação direta, de processo de inferência ou pela aceitação de informações provenientes de outras fontes como amigos, televisão, jornais, livros, etc.

Diante do levantamento de variáveis que influenciam no uso da bicicleta no Brasil e exterior, demonstradas nos tópicos 3 e 4 deste artigo, podemos relacioná-las com as três categorias de crenças da Teoria do Comportamento Planejado e outros constructos, enumerando crenças salientes para pesquisas que pretendem aplicar a Teoria do Comportamento Planejado com intuito de identificar as intenções 


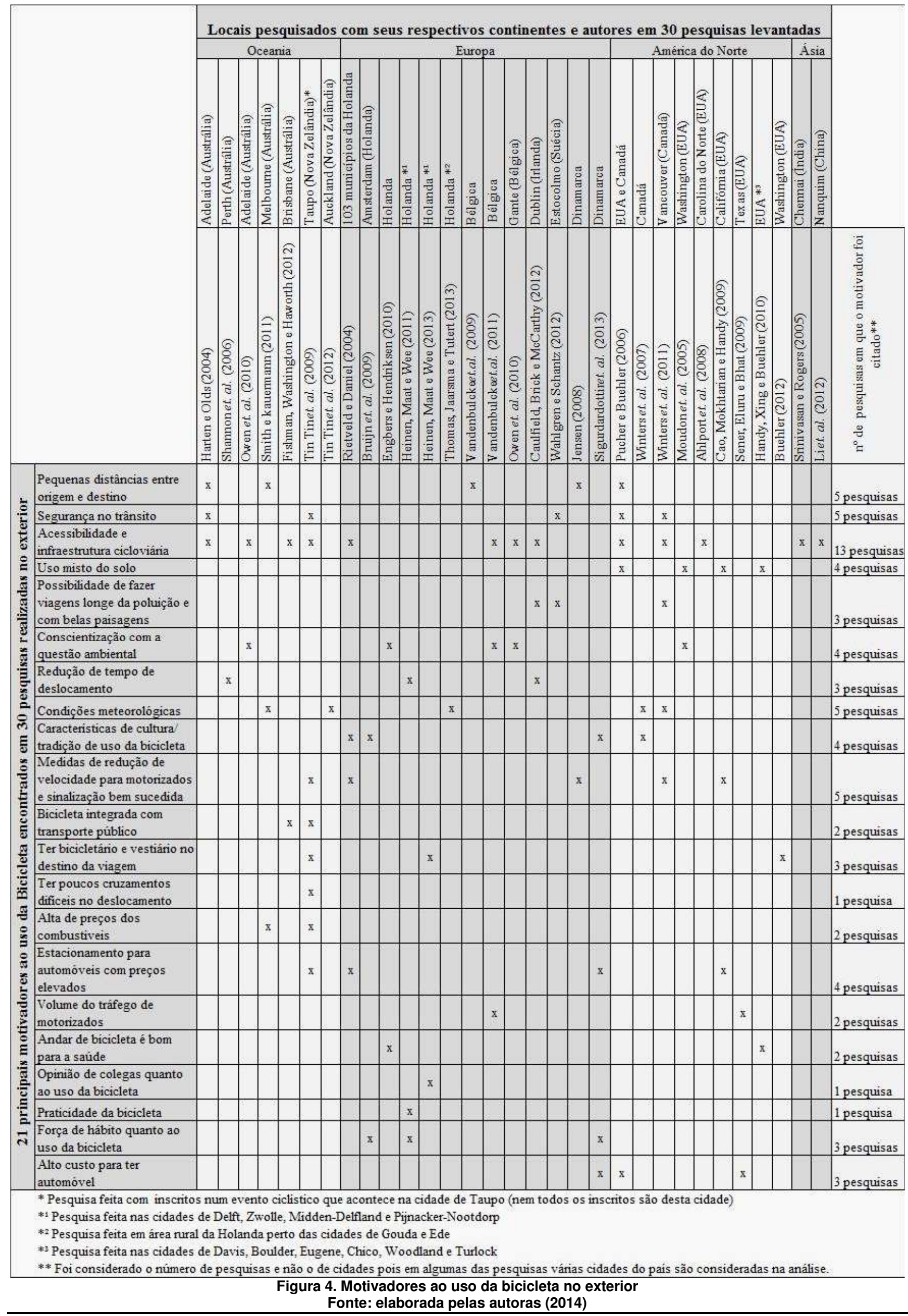




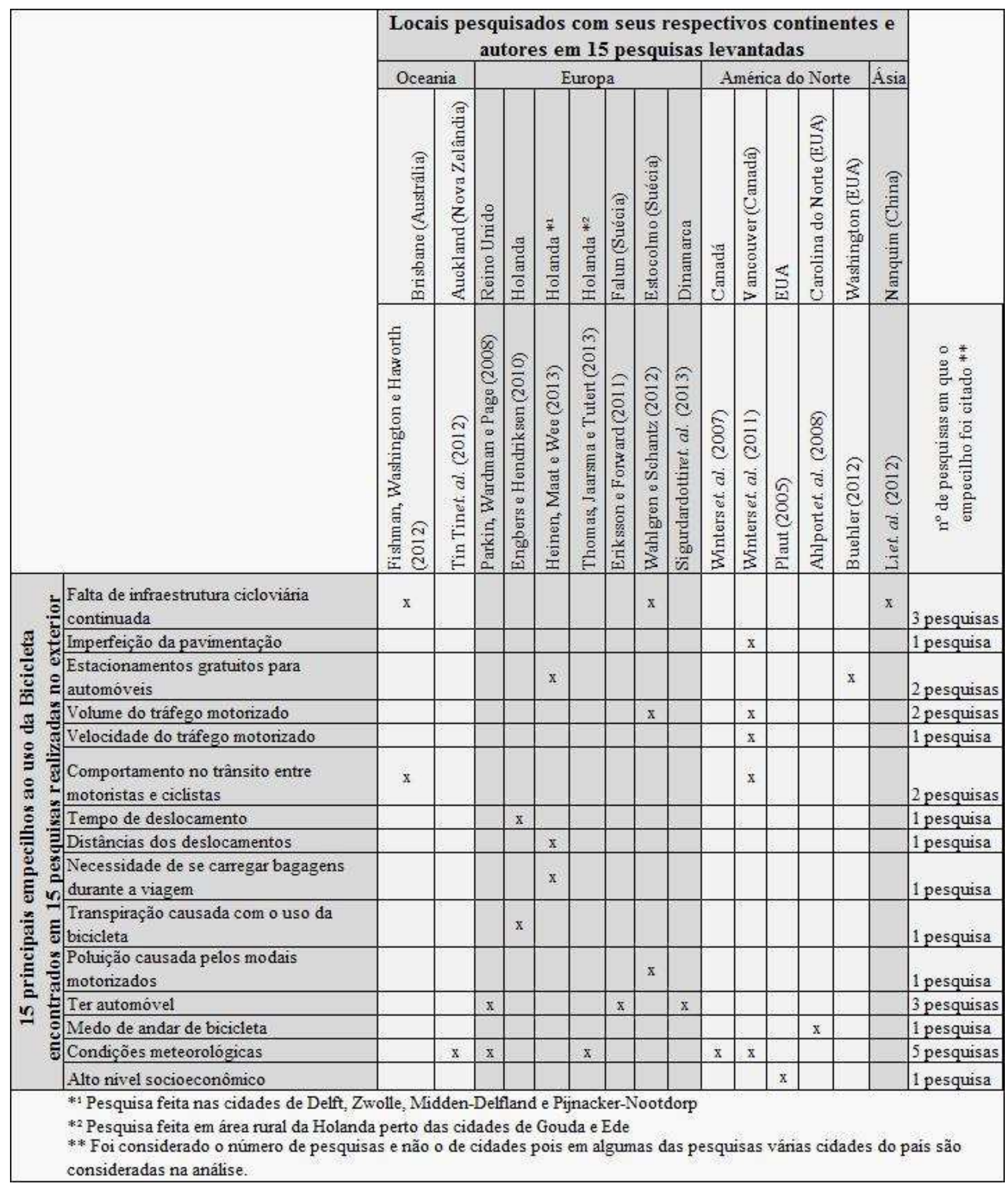

Figura 5. Empecilhos ao uso da bicicleta no exterior

Fonte: elaborada pelas autoras (2014)

de uso da bicicleta. Neste caso, este levantamento seria baseado no processo de inferência e de aceitação de informações proveniente principalmente de pesquisas recentes feitas no Brasil e no exterior.

Depois de levantados diversos empecilhos e motivadores ao uso da bicicleta no Brasil e exterior, 42 variáveis foram identificadas. Dessas 42 variáveis, 36 se enquadram nas características dos três tipos de crenças da Teoria do Comportamento Planejado. As outras 6 variáveis se enquadram em outras categorias, mas podem, inclusive, ser também constructos da Teoria do Comportamento Planejado, já que esta teoria é aberta a inclusão de novos constructos. Esta classificação das variáveis quanto às crenças da Teoria do Comportamento Planejado e a outros constructos foi baseada na observação das 7 pesquisas disponíveis na base SCOPUS de 2000 a 2013 que trabalham esta teoria relacionada ao uso da bicicleta. A seguir, na Figura 6, todas as variáveis levantadas estão relacionadas ao tipo de crença da Teoria do Comportamento Planejado ou a outros tipos de constructos como ocorreu com 6 delas.

Depois de analisar o número de vezes que cada variável foi citada no levantamento feito nas pesquisas nacionais e internacionais, cinco variáveis relacionadas às crenças da Teoria do Comportamento Planejado foram mais mencionadas (no mínimo 6 vezes cada uma) e uma variável relacionada à normas descritivas foi também bastante mencionada (9 vezes). As outras variáveis não chegaram a ser 


\begin{tabular}{|c|c|c|}
\hline $\begin{array}{l}\text { Constructo da Teoria do } \\
\text { Comportamento Planejado }\end{array}$ & $\begin{array}{c}\text { Classificação da crença } \\
\text { relacionada ao } \\
\text { constructo }\end{array}$ & $\begin{array}{c}\text { Variáveis identificadas em pesquisas de } 2000 \text { a } 2013 \text {, } \\
\text { no âmbito nacional e internacional, que influenciam no } \\
\text { uso da bicicleta }\end{array}$ \\
\hline \multirow{15}{*}{$\begin{array}{c}\text { ATITUDE - Consideram a } \\
\text { probabilidade subjetiva de que a } \\
\text { ATITUDE em relação ao } \\
\text { comportamento produza uma } \\
\text { consequência - no caso de } \\
\text { pesquisas em envolvem o } \\
\text { comportamento de uso da } \\
\text { bicicleta, aqui se incluem todas } \\
\text { as crenças que envolvem } \\
\text { caracteristicas da bicicleta. }\end{array}$} & \multirow{15}{*}{$\begin{array}{l}\text { Variáveis que } \\
\text { influenciam no uso da } \\
\text { bicicleta e que são } \\
\text { relacionadas com } \\
\text { Crenças } \\
\text { Comportamentais }\end{array}$} & Praticidade de uso da bicicleta \\
\hline & & Andar de bicicleta é bom para a saúde \\
\hline & & Bem estar em andar de bicicleta \\
\hline & & Conscientização com a questão ambiental \\
\hline & & (Des) Conforto em andar de bicicleta \\
\hline & & Medo de andar de bicicleta \\
\hline & & Vontade de andar de bicicleta \\
\hline & & Sedentarismo \\
\hline & & Local onde reside \\
\hline & & Custo da bicicleta \\
\hline & & (In) Segurança do trânsito \\
\hline & & (In) Segurança pública \\
\hline & & Transpiração causada com o uso da bicicleta \\
\hline & & Necessidade de se carregar bagagens durante a viagem \\
\hline & & Vulnerabilidade do ciclista \\
\hline \multirow{2}{*}{$\begin{array}{l}\text { NORMA SUBJETIVA - refere- } \\
\text { se à percepção das expectativas } \\
\text { que individuos ou grupos têm a } \\
\text { respeito de seus } \\
\text { comportamentos. }\end{array}$} & \multirow{2}{*}{$\begin{array}{l}\text { Variáveis que } \\
\text { influenciam no uso da } \\
\text { bicicleta e que são } \\
\text { relacionadas com } \\
\text { Crenças Normativas }\end{array}$} & (Des) apoio da familia quanto ao uso da bicicleta \\
\hline & & (Des) apoio de colegas quanto ao uso da bicicleta \\
\hline \multirow{23}{*}{$\begin{array}{l}\text { CONTROLE PERCEBIDO } \\
\text { sobre o comportamento é } \\
\text { definido como a crença do } \\
\text { individuo sobre o grau de } \\
\text { facilidade ou de dificuldade para } \\
\text { desempenhar um determinado } \\
\text { comportamento. }\end{array}$} & \multirow{19}{*}{$\begin{array}{l}\text { Variáveis que } \\
\text { influenciam no uso da } \\
\text { bicicleta e que são } \\
\text { relacionadas com } \\
\text { Crenças de Controle }\end{array}$} & Distância do deslocamento \\
\hline & & Tempo de deslocamento com o uso da bicicleta \\
\hline & & Acessibilidade e infraestrutura cicloviária \\
\hline & & Qualidade do serviço de transporte público \\
\hline & & Uso do solo \\
\hline & & Poluição causada pelos modais motorizados \\
\hline & & Preço dos automoveis \\
\hline & & Condições meteorológicas \\
\hline & & Integração da bicicleta com outros modais \\
\hline & & Bicicletários e/ou paraciclos e vestiários \\
\hline & & Cruzamentos no trajeto a ser percorrido \\
\hline & & Preço dos combustiveis \\
\hline & & Estacionamentos para automóveis \\
\hline & & Volume do tráfego motorizado \\
\hline & & Velocidade do tráfego motorizado \\
\hline & & Iluminação \\
\hline & & Pavimentação \\
\hline & & Topografia \\
\hline & & Preço de motocicletas \\
\hline & Outros Constructos & $\begin{array}{l}\text { Variáveis identificadas em pesquisas de } 2000 \text { a } 2013 \text {, } \\
\text { no âmbito nacional e internacional, que influenciam no } \\
\text { uso da bicicleta }\end{array}$ \\
\hline & \begin{tabular}{|} 
Variáveis de norma \\
descritiva (relacionadas \\
à como os outros se \\
comportam em relação \\
ao comportamento)
\end{tabular} & Comportamento no trânsito entre motoristas e ciclistas \\
\hline & $\begin{array}{c}\text { Variáveis } \\
\text { sociodemográficas }\end{array}$ & $\begin{array}{l}\text { Nivel socioeconômico, ter automóvel, ter bicicleta, } \\
\text { cultura e tradição de uso da bicicleta }\end{array}$ \\
\hline & Força do hábito & Força do hábito quanto ao uso da bicicleta \\
\hline
\end{tabular}

Figura 6. Variáveis que influenciam no uso da bicicleta e as crenças e constructos da Teoria do Comportamento Planejado Fonte: elaborada pelas autoras (2014)

mencionadas mais de 4 vezes entre as pesquisas nacionais e internacionais, e não serão consideradas como referência de crenças salientes para outras pesquisas de forma geral.
Entretanto, a baixa frequência de citação de algumas variáveis não desmerece o valor de cada uma, principalmente diante da importância na consideração das caracte- 
rísticas de cada local a ser pesquisado, relativas à infraestrutura urbana, segurança no trânsito e pública, cultura, políticas de mobilidade, clima da cidade, dentre outras. Diante disto, a cada pesquisa que trabalhe a Teoria do Comportamento Planejado e o uso da bicicleta, outras variáveis devem ser observadas para ver se se enquadram como sendo crenças salientes e consequentemente sejam exploradas num questionário padrão da Teoria do Comportamento Planejado.

Segue abaixo a relação das variáveis obtidas a partir do levantamento feito neste artigo que podem ser consideradas como referência de crenças salientes para pesquisas que pretendem usar a Teoria do Comportamento Planejado com intuito de identificar as intenções de uso da bicicleta.

- Duas crenças salientes relacionadas a Crenças Comportamentais (Atitude)

1. 'Andar de bicicleta é bom para a saúde' - Atitudes em relação aos benefícios do ciclismo, como por exemplo, o benefício de ser bom para a saúde, são importantes na opção do indivíduo usar a bicicleta. Esta variável foi a mais citada como motivadora ao uso da bicicleta dentre as pesquisas nacionais nas cidades paulistas de São Carlos e Rio Claro (PROVIDELO e SANCHES, 2010) e São José do Rio Preto (SOUZA, SANCHES e FERREIRA, 2013); Aracajú-SE (ARAÚJO et. al., 2009) e Salvador-BA (RIBEIRO, 2005), e também foi mencionada em duas pesquisas do exterior, as quais foram em várias cidades da Holanda (ENGBERS e HENDRIKSEN, 2010) e em seis cidades dos EUA (HANDY, XING e BUEHLER, 2010).

2. 'Segurança do trânsito' - técnicas de redução de velocidade ou de segregação das vias por tipo de modo de transporte estão entre as formas de garantir segurança para o ciclista e outros usuários do trânsito. Esta variável foi citada como empecilho ao uso da bicicleta em quatorze cidades do Brasil diante da insegurança que os ciclistas sentem ao se deslocarem. Foi citada nas cidades paulistas de Lorena, Piracicaba e Santo André (MIRANDA, 2003), Araçatuba (PEZZUTO e SANCHES, 2004) e São José do Rio Preto (SOUZA, SANCHES e FERREIRA, 2013); Florianópolis-SC (MIRANDA, 2003); Aracajú-SE (ARAÚJO et. al., 2009); Juiz de Fora-MG (CASTANÕN, 2011); Recife-PE (SILVEIRA, 2010); Curitiba-PR (FRANCO, 2011; CAMARGO, 2012); BrasíliaDF (DELABRIDA, 2004); Salvador-BA (RIBEIRO, 2005); Fortaleza-CE (MAIA e MOREIRA, 2010) e Belo Horizonte-MG (PEREIRA, SOUZA e MOREIRA, 2011). Enquanto isto, esta variável foi citada como motivadora ao uso da bicicleta em cinco pesquisas do exterior realizadas na Austrália (HARTEN e OLDS, 2004), Nova Zelândia (TIN TIN et. al., 2009), Suécia (WAHLGREN e SCHANTZ, 2012), Canadá (PUCHER e BUEHLER, 2006; WINTERS et. al., 2011) e EUA (PUCHER e BUEHLER, 2006).

- Três crenças salientes relacionadas a Crenças de Controle

1. 'Tempo de deslocamento com o uso da bicicleta' Dependendo da distância da viagem e das condições do trajeto, este tempo vai variar e interferir na escolha pela bicicleta. Esta variável foi citada como motivadora ao uso da bicicleta em três cidades do Brasil: Teresina-PI (LEITE, 2011), Aracajú-SE (ARAÚJO et. al., 2009) e Salvador-BA (RIBEIRO, 2005) e em três pesquisas do exterior realizadas na Austrália (SHANNON et. al., 2006), Holanda (HEINEN, MAAT e WEE, 2011) e Irlanda (CAULFIELD, BRICK e MCCARTHY, 2012). Esta variável também foi citada como empecilho ao uso da bicicleta numa pesquisa feita na Holanda (ENGBERS e HENDRIKSEN, 2010).

2. 'Condições meteorológicas' - Tem épocas que o clima favorece ao deslocamento por bicicleta e tem épocas que não, mas mesmo em épocas desfavoráveis, se houver infraestrutura cicloviária adequada, este empecilho pode ser reduzido. Esta variável foi citada como empecilho ao uso da bicicleta em uma cidade do Brasil, Curitiba (KIENTEKA, 2012), e em cinco pesquisas do exterior realizadas na Holanda (THOMAS, JAARSMA e TUTERT, 2013), Nova Zelândia (TIN TIN et. al., 2012), Canadá (WINTERS et. al., 2007 e 2011) e Reino Unido (PARKIN, WARDMAN e PAGE, 2008). Com exceção do Reino Unido, todos os outros lugares do exterior que tiveram esta variável como empecilho também a tiveram como motivadora ao uso da bicicleta, pois a variação do clima interferiu nesta posição. Além desses lugares, uma pesquisa realizada em Melbourne, na Austrália (SMITH e KAUERMANN, 2011), também teve 'condições meteorológicas' como variável motivadora ao uso da bicicleta.

3. 'Acessibilidade e infraestrutura cicloviária' - Caso haja adequada infraestrutura e acessibilidade para uso da bicicleta o uso deste modal será bem mais fácil, inclusive, esta variável é uma das que mais influencia neste uso. Esta variável foi citada como empecilho ao uso da bicicleta em nove cidades do Brasil diante da falta de infraestrutura cicloviária adequada nessas cidades. Foi citada nas cidades paulistas de Lorena, Piracicaba e Santo André (MIRANDA, 2003) e Araçatuba (PEZZUTO e SANCHES, 2004); Florianópolis-SC (MIRANDA, 2003); Recife-PE (SILVEIRA, 2010); Curitiba-PR (FRANCO, 2011; CAMARGO, 2012); Fortaleza-CE (MAIA e MOREIRA, 2010) e Belo Horizonte-MG (PEREIRA, SOUZA e MOREIRA, 2011). Enquanto isto, esta variável foi citada como motivadora ao uso da bicicleta em treze pesquisas do exterior realizadas na Austrália (HARTEN e OLDS, 2004; OWEN et. al., 2010; FISHMAN, WASHINGTON e HAWORTH, 2012), na Nova Zelândia (TIN TIN et. al., 2009), na Irlanda (CAULFIELD, BRICK e McCARTHY, 2012), na Índia (SRINIVASAN e ROGERS, 2005), na China (LI et. al., 2012), na Holanda (RIETVELD e DANIEL, 2004), nos EUA (PUCHER e BUEHLER, 2006; AHLPORT et. al.,2008), no Canadá (PUCHER e BUEHLER, 2006; WINTERS et. al.,2011) e na Bélgica (VANDENBULCKE et.al.,2011; OWEN et. al., 2010).

- Uma crença saliente relacionada a Crenças Descritivas 
1. 'Comportamento no trânsito entre motoristas e ciclistas' - conflito ou harmonia relativa à convivência e respeito no espaço viário entre motoristas e ciclistas. Esta variável se enquadra como um constructo adicional da Teoria do Comportamento Planejado. Foi citada como empecilho ao uso da bicicleta em sete cidades do Brasil: nas cidades paulistas de Lorena, Piracicaba e Santo André (MIRANDA, 2003); Florianópolis-SC (MIRANDA, 2003); Juiz de Fora-MG (CASTANÕN, 2011); Curitiba-PR (FRANCO, 2011; CAMARGO, 2012) e Fortaleza-CE (MAIA e MOREIRA, 2010), e como empecilho ao uso da bicicleta em duas pesquisas do exterior realizadas em Brisbane - Austrália (FISHMAN, WASHINGTON e HAWORTH, 2012) e em Vancouver - Canadá (WINTERS et. al., 2011).

\section{CONCLUSÕES}

Neste artigo, variáveis que influenciam no uso da bicicleta, no âmbito nacional e internacional, foram demonstradas e classificadas quanto às crenças e constructos da Teoria do Comportamento Planejado. Com essa classificação ficou possível determinar crenças salientes que podem servir de referência deste tipo de crença quanto ao uso da bicicleta em outras pesquisas que queiram determinar intenções deste uso, facilitando na elaboração de questionário padrão da Teoria do Comportamento Planejado nessas pesquisas. Foram determinadas seis variáveis como sendo crenças salientes, sendo cinco delas relativas às crenças da Teoria do Comportamento Planejado e uma se enquadrando como um novo constructo.

As seis variáveis classificadas como crenças salientes e que podem ser exploradas num questionário padrão da Teoria do Comportamento Planejado em pesquisas que pretendem identificar as intenções de uso da bicicleta foram: (1) 'Andar de bicicleta é bom para a saúde'; (2) 'Tempo de deslocamento com o uso da bicicleta'; (3) 'Condições meteorológicas'; (4) 'Segurança do trânsito'; (5) 'Acessibilidade e infraestrutura cicloviária' e (6) ‘Comportamento no trânsito entre motoristas e ciclistas'.

Esta classificação de seis variáveis como sendo crenças salientes não impede que outras variáveis relacionadas ao uso da bicicleta sejam exploradas num questionário padrão da Teoria do Comportamento Planejado, pois dependendo do local da pesquisa, outras variáveis podem ser relevantes de serem exploradas. Variáveis sociodemográficas, segurança pública, distância do deslocamento, medo de andar de bicicleta e volume do tráfego motorizado, são, por exemplo, fatores que interferem bastante em cidades do Brasil, e que podem ser explorados em pesquisas que pretendem aplicar a TCP relacionada ao uso da bicicleta, pela grande interferência que possuem na intenção de uso deste modal. A classificação de apenas seis crenças salientes não desmerece a importâncias de todas as outras 36 variáveis demonstradas neste artigo, onde poderão ser observadas em outras pesquisas e eventualmente poderão ser consideradas como crenças salientes.

De acordo com Silveira e Maia (2013) a Teoria do Comportamento Planejado se adequa bem em pesquisas que pretendem identificar as intenções que precedem o comportamento relacionado com o uso da bicicleta. Nesse sentido, observa-se que trabalhar o componente comportamento com a aplicação da TCP pode colaborar para um melhor desenho de políticas e medidas que incentivem o uso do transporte sustentável, em particular, a bicicleta.

\section{REFERÊNCIAS}

AHLPORT, K.N.; LINNAN, L.; VAUGHN, A.; EVENSON, K.R.; WARD, D. S. Barriers to and Facilitators of Walking and Bicycling to School: Formative Results From the Non-Motorized Travel Study. Health Education \& Behavior, Vol. 35 (2): 221-244, 2008. DOI: $10.1177 / 1090198106288794$.

AJZEN, I. From intentions to actions: A theory of planned behavior. In J. Kuhl \& J. Beckman (Eds.), Action-control: From cognition to behavior (pp. 11- 39). Heidelberg, Germany: Springer, 1985. DOI: 10.1007/978-3-642-69746-3 2.

AJZEN, I. The Theory of Planned Behavior. Organizational behavior and human decision processes, 50, 179-211, 1991. DOI: 10.1016/0749-5978(91)90020-T.

AJZEN, I. Attitudes, personality, and behavior (2nd. Edition), Milton-Keynes, England: Open University Press / McGraw- Hill, 2005.

AJZEN, I. Behavioral interventions based on the Theory Planned Behavior. Disponível em: <http://people.umass.edu/aizen/pdf/ tpb.intervention.pdf>, acesso em setembro de 2013, 2006.

ARAÚJO, M.R.M.; SOUZA, D.A.; OLIVEIRA, J.M.; JESUS, M.S.; SÁ, N.R.; SANTOS, P.A.C.; MACEDO Jr., R.; LIMA, T.C. Andar de bicicleta: contribuições de um estudo psicológico sobre mobilidade. Temas em Psicologia, vol. 17, n2, 481-495, 2009.

AJZEN, I.; FISHBEIN, M. Attitudes and the attitude-behavior relation: Reasoned and automatic processes. In W. Stroebe \& M. Hewstone (Eds.), European Review of Social Psychology (pp. 133). John Wiley \& Sons, 2000. DOI: 10.1080/14792779943000116.

BRUIJN, G.; KREMERS, S.P.J.; SINGH, A.; PUTTE, B.; MECHELEN, W. Adult Active Transportation - Adding Habit Strength to the Theory of Planned Behavior. American Journal of Preventive Medicine, 36 (3), pp. 189 - 194, 2009. DOI: 10.1016/j.amepre.2008.10.019.

BUEHLER, R. Determinants of bicycle commuting in the Washington, DC region: The role of bicycle parking, cyclist showers, and free car parking at work. Transportation Research Part D, 17, 525-531, 2012. DOI:10.1016/j.trd.2012.06.003.

CAMARGO, E.M. Barreiras e facilitadores para o uso da bicicleta em adultos na cidade de Curitiba - Um estudo com grupos focais. Dissertação de Mestrado. Programa de Pós Graduação em Educação Física. Universidade Federal do Paraná, Curitiba, 2012.

CAO, X.J; MOKHTARIAN, P.L.; HANDY, S.L. The relationship between the built environment and nonwork travel: A case study of Northern California. Transportation Research Part A, 43, 548559, 2009. DOI: 10.1016/j.tra.2009.02.001.

CASTAÑON, U.N. Uma proposta de mobilidade sustentável: o uso da bicicleta na cidade de Juiz de Fora. Dissertação de Mestrado. Programa de Pós-Graduação em Engenharia de Transportes. Universidade Federal do Rio de Janeiro, COPPE, Rio de Janeiro RJ, 2011. 
CASTRO, C.M.S.; BARBOSA, H.M.; OLIVEIRA, L.K. Análise do potencial de integração da bicicleta com o transporte coletivo em Belo Horizonte. Journal of Transport Literature. Vol. 7. n. 2. p. $146-170,2013$. ISSN 2238-1031.

CAULFIELD, B.; BRICK, E.; McCARTHY, O.T. Determining bicycle infrastructure preferences - A case study of Dublin. Transportation Research Part D, 17, 413-417, 2012. DOI: 10.1016/j.trd.2012.04.001.

DELABRIDA, Z.N.C. A imagem e o uso da bicicleta: um estudo entre moradores de Taguatinga. Dissertação de Mestrado. Instituto de Psicologia. Universidade de Brasília. Brasília, DF, 2004.

ENGBERS, L.H.; HENDRIKSEN, I.JM. Characteristics of a population of commuter cyclists in the Netherlands: perceived barriers and facilitators in the personal, social and physical environment. International Journal of Behavioral Nutrition and Physical Activity, 7:89, 2010. DOI: 10.1186/1479-5868-7-89.

ERIKSSON, L.; FORWARD, S.E. Is the intention to travel in a pro-environmental manner and the intention to use the car determined by different factors? Transportation Research Part D, 16, 372-376, 2011. DOI: 10.1016/j.trd.2011.02.003.

FISHMAN, E.; WASHINGTON, S.; HAWORTH, N. Barriers and facilitators to public bicycle scheme use: A qualitative approach. Transportation Research Part F, 15, 686-698, 2012. DOI: 10.1016/j.trf.2012.08.002.

FLEISCHFRESSER, I. Estudo sobre as atitudes dos jovens motoristas de Campo Grande - MS. Dissertação de mestrado em Psicologia. Universidade Católica Dom Bosco - UCDB, Campo Grande - MS, 2005.

FRANCO, C.M.A. Incentivos e empecilhos para a inclusão da bicicleta entre universitários. Dissertação de mestrado em Psicologia. Universidade Federal do Paraná, Curitiba-PR, 2011.

HANDY, S.L.; XING, Y.; BUEHLER, T.H. Factors associated with bicycle ownership and use: a study of six small U.S. Cities. Transportation, 37:967-985, 2010. DOI: 10.1007/s11116-0109269-x.

HARTEN, N.; OLDS T. Patterns of active transport in 11-12 year old Australian children. Australian and New Zealand Journal of Public Health, v.28, n.2, 2004. DOI: 10.1111/j.1467-842X.2004. tb00931.x.

HEIDEMANN, L.A.; ARAUJO, I.S.; VEIT, E.A. Um referencial teórico-metodológico para o desenvolvimento de pesquisas sobre atitude: a Teoria do Comportamento Planejado de Icek Ajzen. Revista electrónica de investigación en educación en ciencias REIEC, ISSN 1850-6666,volume 7, n 8, p. 1-10, 2012.

HEINEN, E.; MAAT, K.; WEE, B. van. The role of attitudes toward characteristics of bicycle commuting on the choice to cycle to work over various distances. Transportation Research Part D, 16, 102-109, 2011. DOI: 10.1016/j.trd.2010.08.010.

HEINEN, E.; MAAT, K.; WEE, B. van. The effect of work-related factors on the bicycle commute mode choice in the Netherlands. Transportation, 40:23-43, 2013. DOI 10.1007/s11116-012-93994.

JENSEN, S.U. How to obtain a healthy journey to school. Transportation Research Part A, 42 , 475 - 486, 2008. DOI: 10.1016/j. tra.2007.12.001.

KIENTEKA, M. Aspectos individuais e ambientais associados ao uso da bicicleta no lazer e no transporte entre adultos de Curitiba
- PR. Dissertação de Mestrado. Programa de Pós-Graduação do departamento de Educação Física. Universidade Federal do Paraná, Curitiba - PR, 2012.

LEITE, P.S. Análise do comportamento de viagens dos usuários de bicicleta em área rural: estudo de caso em área rural de Teresina. Dissertação de Mestrado em Transportes. Universidade de Brasília, Brasília - DF, 2011.

LI, Z.; WANG, W.; LIU, P.; RAGLAND, D.R. Physical environments influencing bicyclists' perception of comfort on separated and on-street bicycle facilities. Transportation Research Part D, 17, 256-261, 2012. DOI: 10.1016/j.trd.2011.12.001.

MAIA, C.A.; MOREIRA, M.E.P. Caracterização dos deslocamentos de ciclistas e fatores que influenciam suas viagens em Fortaleza-CE. XXIV Congresso de Pesquisa e Ensino em Transportes, ANPET, 2010.

MIRANDA, A.C.M. Comparativos de demandas cicloviárias. $14^{\circ}$ Congresso Brasileiro de Transporte e Trânsito. Associação Nacional dos Transportes Públicos - ANTP, Vitória - ES, 2003.

MOUDON, A.V.; CHANAM, L.; CHEADLE, A.D.; COLLIER, C.W.; JOHNSON, D.; SCHMID, T.L.; WEATHER, R.D. Cycling and the built environment, a US perspective. Transportation Research Part D, 10, 245-261, 2005. DOI: 10.1016/j.trd.2005. 04.001.

MOURA, L.R.C.; VEIGA, R.T.; CUNHA, N.R.S.; MOURA, L.E.L.DE. A Teoria do Comportamento Planejado e sua modificação com a inclusão do comportamento passado: um estudo sobre o comportamento de tentar perder peso. Perspectiva, Erechim. v.36, n.136, p.167-179, dezembro, 2012.

MOUTINHO, K.; ROAZZI, A. As teorias da ação racional e da ação planejada: relações entre intenções e comportamentos. Avaliação Psicológica, 9(2), 279 - 287, 2010.

OWEN, N.; BOURDEAUDHUIJ,I.D.; SUGIYAMA, T.;LESLIE, E.; CERIN,E.; DYCK, D.V.; BAUMAN, A. Bicycle Use for Transport in an Australian and a Belgian City: Associations with Built-Environment Attributes. Journal of Urban Health: Bulletin of the New York Academy of Medicine, Vol. 87, No. 2, 2010. DOI: 10.1007/s11524-009-9424-x.

PARKIN, J.; WARDMAN, M.; PAGE, M. Estimation of the determinants of bicycle mode share for the journey to work using census data. Transportation, 35:93-109, 2008. DOI: 10.1007/s11116-007-9137-5.

PEIXOTO, F.C. Estudo do comportamento planejado na escolha da faculdade: uma aplicação ao contexto itabirano. Dissertação de Mestrado em Administração. FUMEC. Belo Horizonte, MG, 2007.

PEREIRA, A.C.C.; SOUZA, A.A.; MOREIRA, D.R. Aceitabilidade do uso da bicicleta como modo de transporte na Universidade Federal de Minas Gerais. XXV Congresso de Pesquisa e Ensino em Transportes, ANPET. Belo Horizonte, MG, 2011.

PEZZUTO, C.C.; SANCHES, S.P. Identificação dos fatores que influenciam no uso da bicicleta. XVIII Congresso de Pesquisa e Ensino em Transportes, ANPET. Florianópolis, SC, 2004.

PLAUT, P.O. Non-motorized commuting in the US. Transportation Research Part D, 10, 347-356, 2005. DOI: 10.1016/j.trd. 2005.04.002. 
PROVIDELO, J.K.; SANCHES, S.P. Percepções de indivíduos acerca do uso da bicicleta como modo de transporte. Transportes. v. XVIII, p. 53-61, 2010

PUCHER, J.; BUEHLER, R. Why Canadians cycle more than Americans: A comparative analysis of bicycling trends and policies. Transport Policy, 13, 265-279, 2006. DOI: 10.1016/j. tranpol.2005.11.001.

RIBEIRO, D.M.S. Inclusão da bicicleta, como modo de transporte alternativo e integrado, no planejamento de transporte urbano de passageiros - o caso de Salvador. Dissertação de Mestrado. Engenharia Ambiental Urbana. Universidade Federal da Bahia. Salvador,BA, 2005.

RIETVELD, P.; DANIEL, V. Determinants of bicycle use: do municipal policies matter? Transportation Research Part A, 38, 531550, 2004. DOI:10.1016/j.tra.2004.05.003.

RODRIGUES, H. A formação da intenção em duas culturas: um estudo com o turismo de aventura. Dissertação de Mestrado em Psicologia Social. Universidade de Brasília. Brasília, DF, 2007.

SENER, I.N., ELURU, N., BHAT, C.R. An analysis of bicycle route choice preferences in Texas, US. Transportation, 36:511539, 2009. DOI: 10.1007/s11116-009-9201-4.

SHANNON, T.; GILES-CORTI, B.; PIKORA, T.; BULSARA, M.; SHILTON, T.; BULL, F. Active commuting in a university setting: Assessing commuting habits and potential for modal change. Transport Policy, 13, 240-253, 2006. DOI:10.1016/j. tranpol.2005.11.002.

SIGURDARDOTTIR, S.B.; KAPLAN, S.; MOLLER, M.; TEASDALE, T.W. Understanding adolescentes' intentions to commute by car or bicycle as adults. Transportation Research Part D, 24, 1 - 9, 2013. DOI: 10.1016/j.trd.2013.04.008.

SILVEIRA, M.O. Mobilidade Sustentável: a bicicleta como meio de transporte integrado. Dissertação de Mestrado. Programa de Pós-Graduação em Engenharia de Transportes. Universidade Federal do Rio de Janeiro, COPPE. Rio de Janeiro, RJ, 2010.

SILVEIRA, M.O., MAIA, M.L.A. Comportamento e uso da bicicleta. XXVII Congresso de Pesquisa e Ensino em Transportes, ANPET. Belém, PA, 2013.

SMITH, M.S.; KAUERMANN, G. Bicycle commuting in Melbourne during the 2000s energy crisis: A semiparametric analysis of intraday volumes. Transportation Research Part B, 45, 18461862, 2011. DOI: 10.1016/j.trb.2011.07.003.

SOUZA, A.A.; SANCHES, S.P.; FERREIRA, M.A.G. Atitudes com relação ao uso da bicicleta - um estudo piloto. XXVII Congresso de Pesquisa e Ensino em Transportes, ANPET. Belém, PA, 2013.

SRINIVASAN, S.; ROGERS, P. Travel behavior of low-income residents: studying two contrasting locations in the city of Chennai, India. Journal of Transport Geography, 13, 265-274, 2005. DOI: 10.1016/j.jtrangeo.2004.07.008.

THOMAS, T.; JAARSMA, R.; TUTERT, B. Exploring temporal fluctuations of daily cycling demand on Dutch cycle paths: the influence of weather on cycling. Transportation, 40:1-22, 2013. DOI: $10.1007 / \mathrm{s} 11116-012-9398-5$.

TIN TIN, S.; WOODWARD, A.; THORNLEY, S.; LANGLEY, J.; RODGERS, A.; AMERATUNGA, S. Cyclists' attitudes toward policies encouraging bicycle travel: findings from the Taupo Bicycle Study in New Zealand. Health Promotion International, Vol. 25, No. 1, 2009. DOI:10.1093/heapro/dap041.

TIN TIN, S.; WOODWARD, A.; ROBINSON, E.; AMERATUNGA, S. Temporal, seasonal and weather effects on cycle volume: an ecological study. Environmental Health, 11:12, 2012. DOI: $10.1186 / 1476-069 \mathrm{X}-11-12$

VANDENBULCKE, G.; THOMAS, I.; GEUS, B. de; DEGRAEUWE, B.; TORFS, R.; MEEUSEN, R.; PANIS, L.I. Mapping bicycle use and the risk of accidents for commuters who cycle to work in Belgium. Transport Policy, 16, 77-87, 2009. DOI: 10.1016/j.tranpol.2009.03.004.

VANDENBULCKE, G.; DUJARDIN, C.; THOMAS, I.; GEUS, B. de; DEGRAEUWE, B.; MEEUSEN, R.; PANIS, L.I. Cycle commuting in Belgium: Spatial determinants and 're-cycling' strategies. Transportation Research Part A, 45, 118-137, 2011. DOI: 10.1016/j.tra.2010.11.004.

VASCONCELOS, E.A. Transporte urbano nos países em desenvolvimento: reflexões e propostas. 3ed. São Paulo: Annablume, 2000.

WAHLGREN, L.; SCHANTZ, P. Exploring bikeability in a metropolitan setting: stimulating and hindering factors in commuting route environments. BMC Public Health, 12:168, 2012. DOI: 10.1186/1471-2458-12-168.

WINTERS, M.; FRIESEN, M.C.; KOEHOORN, M.; TESCHKE, K. Utilitarian Bicycling: A Multilevel Analysis of Climate and Personal Influences. American Journal of Preventive Medicine, 32(1), 52-58, 2007. DOI: 10.1016/j.amepre.2006.08.027.

WINTERS, M.; DAVIDSON, G.; KAO, D.; TESCHKE, K. Motivators and deterrents of bicycling: comparing influences on decisions to ride. Transportation, 38:153-168, 2011. DOI: 10.1007/s11116-010-9284-y. 